\title{
BACKGROUND PARENCHYMAL ENHANCEMENT IN MRI EXAMINATION AND ITS CORRELATION WITH MAMMOGRAPHIC BREAST DENSITY AND CLINICAL AND TUMORAL CHARACTERISTICS
}

Yedda Nunes Reis¹, Bruna Salani Mota¹, Carlos Shimizu¹, Marcos Desiderio Ricci¹, Natalia Paula Cardoso¹, Edmund Chada Baracat ${ }^{1}$, José Roberto Filassi ${ }^{1}$

${ }^{1}$ Instituto do Câncer do Estado de São Paulo, Faculdade de Medicina, Universidade de São Paulo - São Paulo (SP), Brazil.

Objective: The aim of this study is to investigate the correlation between background parenchymal enhancement (BPE) in magnetic resonance imaging (MRI) examination and mammographic breast density (MBD) and also the association of BPE with clinical and tumoral characteristics. Methodology: The post hoc analysis from a prospective database (BREASTMRI trial) was performed. Patients with breast cancer stages 0 to III for breast-conserving surgery, from November 2014 to October 2018, were selected. All patients were evaluated with triple assessment and stratified by MBD. Then, they were randomized on a 1:1 basis in two groups whether to perform breast MRI. BPE was classified into four categories according to the ACR BI-RADS MRI (minimal, mild, moderate, and marked). The MBD was also classified according to the ACR BI-RADS (A, B, C, and D). Results: A total of 217 patients were included. The mean age was 57.4 years (33.7-81.7, SD 10.8). Of these, 25 (11.5\%) patients had ductal carcinoma in situ (DCIS) and $192(88.5 \%)$ had invasive breast cancer. The MBD classification was 12 (5.5\%) for A, 93 (42.9\%) for B, 99 (45.6\%) for C, and 13 (6\%) for D. The BPE classification was 105 (48.4\%) minimum, 78 (35.9\%) mild, 29 (13.4\%) moderate, and 5 (2.3\%) marked. Both MBD and BPE were similar in 63 (29\%) of 217 patients and differed in 154 (71\%) of 217 patients. There is no correlation between MBD and BPE (Spearman's rank of 0.240, $\mathrm{p}<0.001$ ). Minimum/mild BPE breasts were more frequent in postmenopausal women ( $\mathrm{p}=0.01)$. B3 lesions were identified significantly higher in moderate/marked BPE breasts $(\mathrm{p}=0.04)$. The median lesion size in breast MRI for minimum/mild BPE breasts was $2.2 \mathrm{~cm}$, and the pathological median size was $2.0 \mathrm{~cm}(\mathrm{p}=0.001,95 \% \mathrm{CI})$. Conclusion: The background parenchymal enhancement does not correlate with MBD.

Keywords: Background Parenchymal Enhancement; Breast Density; Breast MRI; Mammography; Breast Cancer. 